\title{
Spontaneous Round-Shaped Left Atrial Hematoma
}

\author{
Keisuke Kawaida, MD ${ }^{1}$ Yukinori Moriyama, MD ${ }^{1} \quad$ Yoshihiro Fukumoto, MD ${ }^{1} \quad$ Takayuki Ueno, MD ${ }^{1}$ \\ ${ }^{1}$ Division of Cardiovascular Surgery, National Hospital Organization, \\ Kagoshima Medical Center, Kagoshima, Japan \\ Address for correspondence Yukinori Moriyama, MD, 8-1 Shiroyama- \\ Cho, Kagoshima, 892-0853, Japan \\ Int J Angiol 2015;25:e145-e146. \\ (e-mail: mori930@po.synapse.ne.jp).
}

\begin{abstract}
Spontaneously occurring left atrial hematomas are very rare and most of them followed acute clinical course due to hemodynamic deterioration. We presented a case of gradually developing hematoma protruding into the left atrial cavity as a round mass,

Keywords

- cardiac surgery

- cardiovascular disease

- ultrasound

- excision

- hemodynamics

- repair which was completely encapsulated with intact endocardial wall. Emergency surgery was successfully performed. Histopathological study demonstrated subendocardialaged hematoma with papillary endothelial hyperplasia.
\end{abstract}

Left atrial intramural hematoma is a very rare entity and has often been caused by some mechanical factors such as cardiac surgery, catheter intervention, chest trauma, and so on. We report herein a case of spontaneously occurring left atrial hematoma that protruded into the cardiac cavity as a completely encapsulated mass.

\section{Case Report}

A 52-year-old woman was admitted to our hospital with progressive dyspnea and chest pain over a 3-month period. She had no history of cardiothoracic surgery, chest trauma, hemorrhagic disorders, and so on. Her breathing difficulty was aggravated by taking a left decubitus position. The electrocardiogram showed normal sinus rhythm with negative $T$ waves in $V_{1-4}$. Chest roentgenogram revealed signs of remarkable lung congestion. Transthoracic echocardiography demonstrated a slightly mobile round echolucent mass measuring $30 \times 35 \mathrm{~mm}$, which occupied the almost entire left atrial cavity. The mass attached to the posterior left atrial wall, extending close to the mitral orifice, interfered blood flow into the left ventricle ( $\boldsymbol{- F i g . 1}$ ). The patient also had moderate degree of mitral regurgitation with severe pulmonary hypertension. Emergency cardiac surgery was performed via a median sternotomy under a presumptive diagnosis of tumor or thrombus. A moderate amount of serous effusion was found in the pericardial cavity. Cardiopulmonary bypass was established via ascending aorta and bicaval cannulation. Myocardial protection was accomplished with antegrade cold blood potassium cardioplegia. Left atriotomy through Waterson's groove demonstrated a spherical soft sessile tumor, which was excised along with a rim of surrounding atrial wall. The tumor was completely encapsulated with the intact endocardial wall (-Fig. 2). The pulmonary veins were not involved and no tear was found in the left atrial wall. The tissue defect created by tumor removal was repaired with a fresh autologous pericardial patch. Aortic cross-clamp and perfusion times were 62 and 94 minutes, respectively. The excised tumor was filled with sanguineous fluid with some thrombus (-Fig. 3). Pathologic examination confirmed subendocardial-aged hematoma with papillary endothelial hyperplasia. No specific change such as endocarditis, hemangioma, myxoma, and amyloidosis were observed. The postoperative course was uneventful and the repeat echocardiography before discharge showed a normal left atrium without residual hematoma. The patient has shown no sign of recurrence and remains well 6 months after the operation.
DOI http://dx.doi.org/ 10.1055/s-0035-1558428. ISSN 1061-1711. 


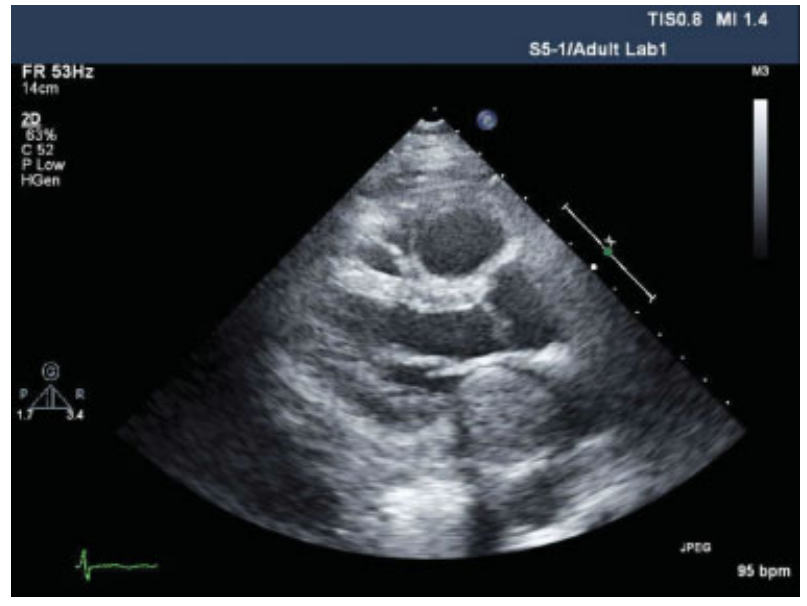

Fig. 1 Transthoracic echocardiography demonstrated a slightly mobile round echolucent mass measuring $30 \times 35 \mathrm{~mm}$, which attached to the posterior left atrial wall and interfered blood flow into the left ventricle.

\section{Discussion}

Left atrial hematomas are usually related to several causative factors including cardiac surgery, trauma, active endocarditis, amyloidosis, hemangioma, or aortic dissection..$^{1-3}$ Concomitant use of anticoagulant and antiplatelet agent may also have an impact on hemorrhagic manifestation. ${ }^{4}$ Meanwhile, spontaneously occurring left atrial hematomas with no such factors as mentioned earlier have been rarely reported in the literatures. ${ }^{5}$ They were all alike in the clinical course of

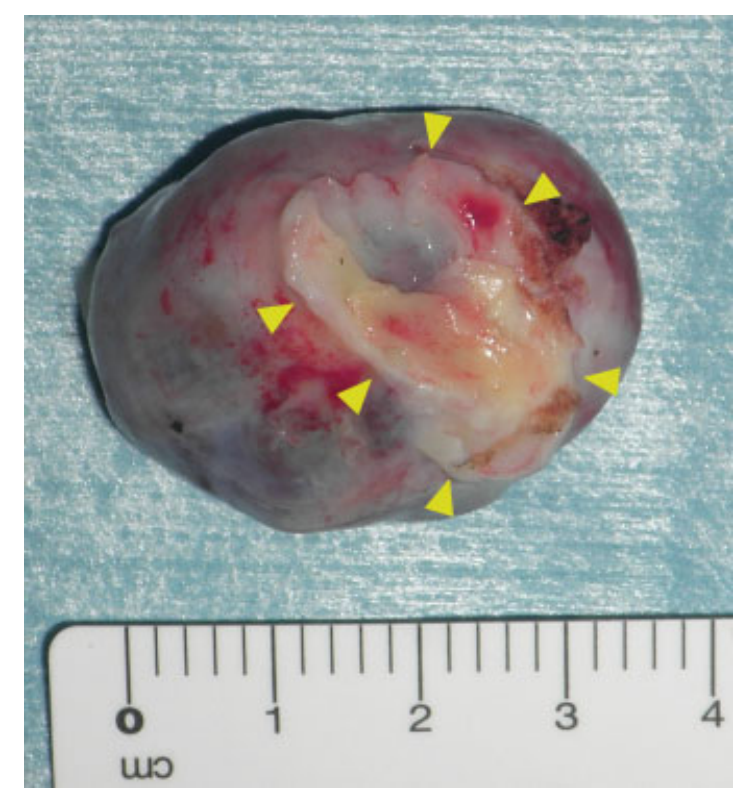

Fig. 2 A spherical soft sessile tumor was excised along with a rim of surrounding atrial wall. Arrows showed attached site to the left atrial wall. The tumor was completely encapsulated with intact endocardial wall.

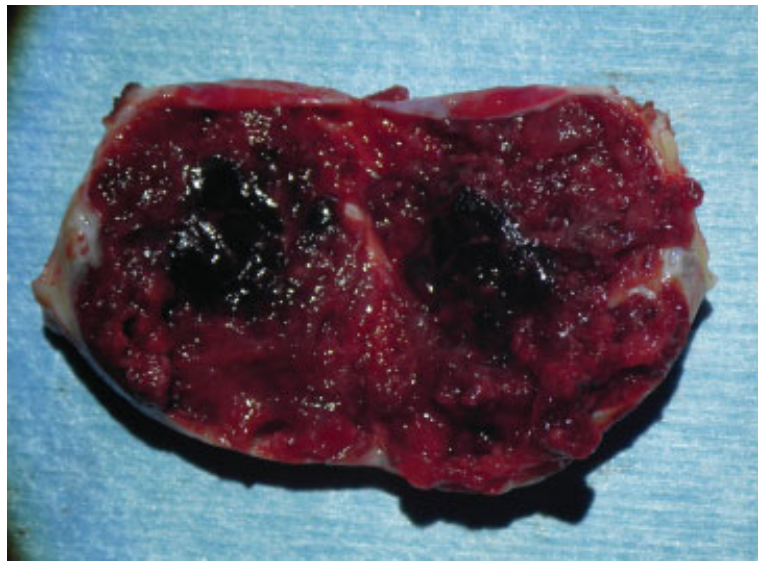

Fig. 3 The excised tumor was filled with sanguineous fluid with some thrombus.

acute hemodynamic deterioration and the expanded hematomas located exclusively in the intramural space, not in the intracardiac cavity. In our case, no obvious cause was found, but the left atrial hematoma gradually grew into the overlying cardiac cavity as a completely round mass covered with intact endocardial wall, which showed quite different morphologic features from the previously reported hematomas. Transthoracic echocardiography was not useful for the differential diagnosis in this case, although transesophageal echocardiography may have been valuable to improve accuracy of diagnosis. As seen in other case report, ${ }^{5}$ however, even if high resolution imaging techniques are applied, it seems very difficult to establish the right diagnosis for this type of atrial mass.

\section{Disclosures}

The author has nothing to disclose with regard to commercial support.

\section{References}

1 Cresce GD, Peluso D, Panfili M, et al. Left atrial wall hematoma as a consequence of percutaneous coronary angioplasty. Ann Thorac Surg 2012;93(3):e57-e59

2 Bruegger D, Sadoni S, Primaychenko M, et al. Conservative treatment of a left atrial intramural hematoma after left atrial thrombus resection and concomitant mitral valve replacement-case report. J Cardiothorac Surg 2011;6:50

3 Lanfranchi A, Gelpi G, Rossi RS, Lemma M. A fast-growing obstructive left atrial intramural hematoma causing acute prolonged chest pain. Interact Cardiovasc Thorac Surg 2009;9(2):363-365

4 Watanabe K, Miguel B, Kemeny JL, Citron B, Camilleri LF. Spontaneous intramural left atrial hematoma associated with systemic amyloidosis. Ann Thorac Surg 2001;72(6):2132-2134

5 Lombardo A, Luciani N, Rizzello V, et al. Images in cardiovascular medicine. Spontaneous left atrial dissection and hematoma mimicking a cardiac tumor: findings from echocardiography, cardiac computed tomography, magnetic resonance imaging, and pathology. Circulation 2006;114(7):e249-e250 